\title{
Frailty and Home Confinement during the COVID-19 Pandemic: Results of a Pre-Post Intervention, Single Arm, Prospective and Longitudinal Pilot Study
}

\author{
C.P. Launay ${ }^{1,2,3}$, L. Cooper-Brown ${ }^{2,3}$, V. Ivensky ${ }^{2,4}$, O. Beauchet ${ }^{1,2,4,5,6,7}$
}

1. Department of Medicine, Division of Geriatric Medicine, Sir Mortimer B. Davis - Jewish General Hospital and Lady Davis Institute for Medical Research, McGill University, Montreal, Quebec, Canada; 2. Centre of Excellence on Longevity of McGill Integrated University Health and Social Services Network, Quebec, Canada; 3. Faculty of Medicine, McGill University, Montreal, Quebec, Canada; 4. Faculty of Medicine, University of Montreal, Montreal, Quebec, Canada; 5. Departments of Medicine and Geriatrics, University of Montreal, Montreal, Quebec, Canada; 6. Research Center of the Geriatric University institute of Montreal, Montreal, Quebec, Canada; 7. Lee Kong Chian School of Medicine, Nanyang Technological University, Singapore.

Corresponding Author: Cyrille Launay, MD, PhD; Department of Medicine, Division of Geriatric Medicine, Sir Mortimer B. Davis - Jewish General Hospital, McGill University, 3755 chemin de la Côte-Sainte-Catherine, Montréal, QC H3T 1E2, Canada; E-mail: cyrille.launay@mcgill.ca; Phone: (+1) 514-340-8222, \#23837; Fax: (+1) 514-340-7547

Keywords: Aged, epidemiology, communitydwelling, frailty, COVID-19.

\section{Dear Editor,}

$\mathrm{T}$ The COVID-19 pandemic had severe consequences for older adults. First, COVID-19 was associated with more severe medical complications and an increased mortality rate in older compared to younger adults (1). Second, home confinement, an intervention that reduces the spread of COVID-19, was associated with adverse consequences for the older community-dwelling population (2). It broke down social networks and the continuum of primary care, resulting in medication or food delivery issues, psychological fallout and increasing frailty risks (3). Frailty assessment provides insight into the degree of older community dwellers' health status vulnerability, social isolation and adverse health event risks, and it should be assessed before interventions are proposed (3). We designed a short assessment tool known as "Evaluation SOcio-GERiatrique" (ESOGER) for Montreal's homebound community-dwelling older adults (3). In a phone call, ESOGER briefly assessed frailty and social isolation and provided recommendations, facilitating contact with health or social care providers who initiate appropriate health and social care plans (3). This study aims to examine the longitudinal effects of ESOGER on frailty and social isolation in Montreal's homebound community-dwelling older adults.

Adopting a pre-post intervention, single arm, prospective and longitudinal design, this experimental study enrolled 119 community-dwelling older adults ( $70.0 \%$ female) for a mean follow-up period of 23.2 \pm 13.6 days. Selection criteria were being age 70 and over, being homebound, living in Montreal, not being involved in an experimental study and agreeing to participate. The assessments (i.e., baseline and follow-up) were performed using ESOGER. ESOGER assessed frailty using the 6-item brief geriatric assessment (BGA), with scores ranging from 0 (no frailty) to 14 (severe frailty) (4), as well as social isolation through accessibility to essential services (i.e., medication and food delivery, home care) and contact Received December 30, 2020 Accepted for publication February 2, 2021 with individuals (family, neighbours, friends, healthcare or social professionals) over the phone or in person. When services and/or social contact were absent, ESOGER provided recommendations addressing social isolation and/or care disruption. Evaluated outcomes were: 1) the difference between baseline and follow-up BGA scores (/14) calculated as (followup score - baseline score) / ((follow-up score + baseline score) /2);2) new-onset moderate to severe frailty staging defined by scores $\geq 5 / 14$ at follow-up, but not baseline, assessment; and 3) unsuccessful or successful implementation of ESOGER recommendations, using two proxies: a) new-onset social isolation and care disruption (i.e., no social isolation and no care disruption at baseline assessment with social isolation and care disruption at follow-up assessment) when ESOGER recommendations were not implemented; or b) addressed social isolation and care disruption (i.e., social isolation and care disruption at baseline assessment with no social isolation and no care disruption at follow-up assessment) when ESOGER recommendations were implemented. Linear and logistic regressions were used to examine the association between frailty (mean BGA score and moderate-to-severe frailty stage used as dependent variables in separate models) and ESOGER recommendations (used as independent variables) adjusted to participants' baseline characteristics (Age $\geq 85$, sex, polypharmacy, length of follow-up). P-values less than 0.05 were considered statistically significant. All statistics were performed using SPSS (version 24.0; SPSS, Inc., Chicago, IL). The protocol received Jewish General Hospital (McGill University, Quebec, Canada) Research Ethics Committee approval.

Baseline characteristics of participants are shown in the Table 2. In our study, social isolation decreased significantly from baseline to follow-up assessments (62.2\% versus $38.7 \%$ with $\mathrm{P} \leq 0.001$ ), whereas frailty increased significantly (BGA score $4.1 \pm 3.2$ and moderate-to-severe frailty $47.9 \%$ versus BGA score 5.1 \pm 3.5 and moderate-to-severe frailty $58.0 \%$ with $\mathrm{P} \leq 0.001)$. As shown in Table 1, the implementation of ESOGER recommendations was not associated with significant variations in frailty $(\mathrm{P}>0.166)$, whereas the absence of the implementation of ESOGER recommendations was associated with a significant increase in frailty $(\mathrm{P} \leq 0.038)$. 
Table 1. Multiple linear and regression models showing the association between frailty (mean BGA score and moderate-to-severe frailty stage used as separated dependent variables) and ESOGER recommendations (used as independent variables), adjusted for participants' baseline characteristics $(n=119)$

\begin{tabular}{|l|c|c|c|c|c|c|}
\hline ESOGER recommendations implemented & \multicolumn{3}{|c|}{ Brief geriatric assessment score variation* } & \multicolumn{3}{|c|}{ Moderate-to-severe frailty $\dagger$} \\
\hline & $\mathbf{B}$ & {$[\mathbf{9 5 \%} \mathbf{C I}]$} & P-Value & OR & {$[\mathbf{9 5 \%}$ CI] } & P-Value \\
\hline No & 57.5 & {$[3.1 ; 111.9]$} & $\mathbf{0 . 0 3 8}$ & 9.5 & {$[1.6 ; 57.9]$} & $\mathbf{0 . 0 1 4}$ \\
Yes & -19.3 & {$[-46.7 ; 8.1]$} & 0.166 & 0.7 & {$[0.1 ; 3.5]$} & 0.632 \\
\hline
\end{tabular}

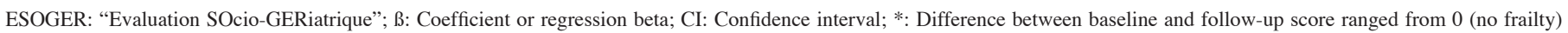

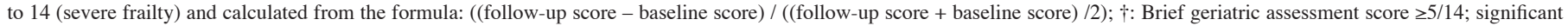
P-values (i.e., $\leq 0.05$ ) in bold.

Table 2. Baseline characteristics of participants $(n=119)$

\begin{tabular}{|c|c|c|}
\hline Characteristics & Values & {$[95 \% \mathrm{CI}]$} \\
\hline Age $\geq 85, \mathrm{n}(\%)$ & $50(42.0)$ & {$[33.0-51.0]$} \\
\hline Male, $\mathrm{n}(\%)$ & $36(30.3)$ & [21.9-38.6] \\
\hline Living alone, $\mathrm{n}(\%)$ & $76(63.9)$ & {$[55.1-72.6]$} \\
\hline Social isolation*, n (\%) & $74(62.2)$ & {$[53.3-71.0]$} \\
\hline Polypharmacy $\dagger, \mathrm{n}(\%)$ & $49(41.2)$ & {$[32.2-50.2]$} \\
\hline Walking aid, n (\%) & $52(43.7)$ & [34.7-52.7] \\
\hline Temporal disorientation $\neq, \mathrm{n}(\%)$ & $7(5.9)$ & [1.6-10.2] \\
\hline \multicolumn{3}{|l|}{ 6-item BGA } \\
\hline Score $(/ 14)$, mean \pm SD & $4.1 \pm 3.2$ & {$[3.5-4,7]$} \\
\hline Frailty $\S, \mathrm{n}(\%)$ & $57(47.9)$ & {$[38.8-57.0]$} \\
\hline Length of follow-up (day), mean \pm SD & $23.2 \pm 13.6$ & [20.7-25.7] \\
\hline
\end{tabular}

BGA: brief geriatric assessment; SD: standard deviation; CI: Confidence interval; *: no formal (i.e., health and/or social professional) or informal (i.e., family and/or friends) help; $\uparrow$ : inability to give the current year and/or month; $\$$ : Number of different medications taken daily $\geq 5$; $\S$ : Score 6 -item BGA $>6$ with score ranging from 0 (no frailty) to 14 (severe frailty)

Home confinement was associated with increased frailty in the studied sample of older community dwellers, and this change in frailty was dependent on ESOGER recommendation implementation. There was a significant increase in frailty when ESOGER recommendations were not implemented, whereas no change was observed when they were implemented. The negative impact of social isolation on older adults' health condition has previously been reported $(5,6)$. This effect could explain the increased frailty observed among participants in this study for whom ESOGER recommendations were not implemented. The absence of significant changes to frailty when ESOGER recommendations were implemented shows that telemedicine may be an effective approach to sustaining the continuum of care in vulnerable homebound patients during crises like COVID-19. The main limitation of our study is the small sample size of participants, and thus studies recruiting greater number of participants are needed to confirm the results of our pilot study. In addition, further developing such interventions may help to remotely stabilize patients and to avoid seeing them in consultation.

Conflicts of Interest: None declared by the authors.

\section{References}

1. Du RH, Liang LR, Yang CQ, Wang W, Cao TZ, Li M, Guo GY, Du J, Zheng CL, Zhu Q, Hu M, Li XY, Peng P, Shi HZ. Predictors of mortality for patients with COVID-19 pneumonia caused by SARS-CoV-2: a prospective cohort study. Eur Respir J. 2020;55(5):2000524.

2. https://blogs.bmj.com/bmj/2020/06/15/covid-19-will-be-followed-by-adeconditioning-pandemic/ (July 07, 2020 date last accessed)

3. Beauchet O, Cooper-Brown L, Ivensky V, Launay CP. Telemedicine for housebound older persons during the Covid-19 pandemic. Maturitas 2020 [Epub ahead of print]

4. Launay CP, de Decker L, Kabeshova A, Annweiler C, Beauchet O. Screening for older emergency department inpatients at risk of prolonged hospital stay: the brief geriatric assessment tool. PLoS One. 2014;9(10): e110135.

5. Brooke J, Jackson D. Older people and COVID-19: Isolation, risk and ageism. J Clin Nurs. 2020;29(13-14): 2044-2046

6. Kf B, Jl H, Ta D. Preventing Frailty Progression during the COVID-19 Pandemic. J Frailty Aging. 2020;9(3):130-131

How to cite this article: C.P. Launay, L. Cooper-Brown, V. Ivensky, et al. Frailty and Home Confinement during the COVID-19 Pandemic: Results of a Pre-Post Intervention, Single Arm, Prospective and Longitudinal Pilot Study. J Frailty Aging 2021;10(4)361-362; http://dx.doi.org/10.14283/jfa.2021.9 